\title{
Unequal Priority Arrangement for Delivering Streaming Videos over Differentiated Service Networks
}

\author{
Chu-Chuan Lee ${ }^{1}$, Pao-Chi Chang², and Shih-Jung Chuang ${ }^{2}$ \\ ${ }^{1}$ Chunghwa Telecommunication Laboratories, R.O.C. \\ ${ }^{2}$ Department of Communication Engineering, National Central University, R.O.C. \\ \{cclee, pcchang, sjchuang\}@vaplab.ee.ncu.edu.tw
}

\begin{abstract}
Video packets have different significances due to the video coding property. When delivering video data without priority strategy over the Internet, it will seriously degrade the received picture quality. This paper proposes an Unequal Priority Arrangement (UPA) mechanism for video transmission on differentiated service networks. UPA determines the priority of a video packet according to the evaluation from both temporal and spatial domains simultaneously. From the temporal domain, UPA evaluates the packet significance based on the temporal position of the packet and the induced error propagation if the packet is lost. From the spatial domain, UPA evaluates the packet importance based on its content, where the ratio of intra-refreshed MBs is used. Moreover, according to the video complexity, UPA can flexibly adjust the weight based on the evaluation results from temporal and spatial domains. Simulation results show that delivering video data with UPA on differentiated service network outperforms traditional temporal-based and spatial-based priority strategies up to $1.8 \mathrm{~dB}$ and $1 \mathrm{~dB}$, respectively.
\end{abstract}

Keywords: Differentiated services, streaming videos, unequal priority arrangement.

\section{Introduction}

Most video coding methods exploit both temporal and spatial redundancies to reduce required transmission rate and achieve high compression efficiency. In the spatial domain, there exists a high correlation within a picture. In the temporal domain, there usually exists a high similarity between successive pictures. However, the received video quality is highly sensitive to packet loss. When a video packet that belongs to I-frame is lost due to network congestion, all frames belonging to the same GOP (Group of Picture) are hurt due to error propagation in the decoding process. This phenomenon causes significant degradation of received picture quality. Moreover, all succeeding frames belonging to the same GOP are also hurt if a video packet that belongs to P-frame is lost, as shown in Fig. 1. Therefore, a robust network mechanism that can provide sufficient protections to video data is essential for received picture quality.

Unfortunately, the default QoS (Quality of Service) strategy of Internet is the besteffort transmission, which is lack of QoS guarantee to encoded video data. In order to enhance the QoS capability of Internet, the Internet Engineering Task Force (IETF) 
had proposed two QoS approaches: Integrated Services (InteServ) [1] and Differentiated Services (DiffServ) [2]. InteServ can provide the flow-based QoS guarantee to delivered data. However, InteServ has the scalability problem due to its high implementation complexity. DiffServ was thus proposed to solve the above scalability problem and was popularly implemented in network equipments. In DiffServ, each packet is assigned and classified to one of few classes. However, if much video data with the same class arrive to a network equipment simultaneously, DiffServ cannot provide the absolute delay and loss guarantees to each video flow. That is, the congestion loss and delay tolerance violation of video packets are still possible to happen even the DiffServ network is implemented. DiffServ can only provide the class-based QoS guarantee to delivered data, which is not sufficient to requirements of compressed video traffic.

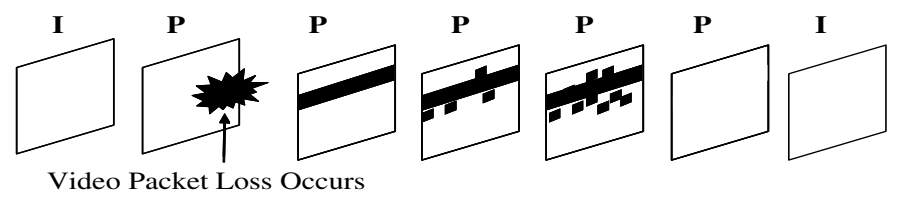

Fig. 1. Error propagation effect if a video packet of P-frame is lost

To prevent the unexpected packet loss of significant video frames such as I-frames in DiffServ network, an unequal priority assignment scheme is required for video packets at the video sender side. The priority of video packet also implies the distortion effect induced by packet loss. Many research results were developed in past few years. In [3] and [4], the frame type (I-/P-/B-frame) is directly used to classify the priorities of video packets. Video packets that belong to I-frame and B-frame always have the highest and the lowest priorities, respectively. However, the error propagation effect of each packet in the temporal domain is not considered. In [5], the error propagation influence of each packet is estimated at the sender side according to its temporal position in a GOP. Two packets that belong to different P-frames have different priorities. However, the method in [5] still ignored the content diversity among these video packets that belong to the same video frame. In [6], the technique of intra-refreshed MacroBlock (MB) is used to alleviate error propagation. The content of each packet is evaluated for determining the packet significance in the spatial domain. According to the ratio of the number of intra-refreshed MBs to the total number of MBs in a packet, the method of [6] defined the Quality Enhancement (QE) of a given video packet. However, the error propagation effect of each packet in the temporal domain is not examined in [6].

This study proposes an Unequal Priority Arrangement (UPA) mechanism for video transmission on differentiated service networks. In contrast to traditional temporalbased or spatial-based priority assignment methods [5][6], UPA determines the priority of a video packet according to the evaluation from both temporal and spatial domains simultaneously. From the temporal domain, UPA evaluates the packet significance based on the temporal position of the packet and the induced error propagation if the packet is lost. From the spatial domain, UPA evaluates the packet importance based on its content, where the ratio of intra-refreshed MBs is used. Moreover, according to the video complexity, UPA can flexibly adjust the weight 
based on the evaluation results from temporal and spatial domains. Regarding a video sequence with low motion variation and low complexity, the evaluation from spatial domain dominates the packet priority. In contrast, the evaluation from temporal domain dominates the packet priority if a video sequence has high motion variation and high complexity.

The remaining of this paper is organized as follows. The detailed process of UPA is presented in Section 2. Simulation environment and results are discussed in Section 3. Finally, Section 4 concludes this paper.

\section{Unequal Priority Arrangement Strategy}

\subsection{Determining Significance Grade of Video Packets}

Since a lost video packet affects the received picture quality from the temporal and spatial domain simultaneously, UPA integrates and improves the methods utilized in [5] and [6]. The $i$-th packet of the $k$-th video frame in a GOP is denoted as $\operatorname{VP}_{k i}$. This study then estimates the Significance Grade (SG) of $\mathrm{VP}_{k i}$ as

$$
\begin{aligned}
S G_{k i} & =(1-\alpha) \cdot S G T_{k i}+\alpha \cdot S G S_{k i} \\
& =(1-\alpha)\left(\sqrt{\left(N_{I}-k+1\right) \cdot Q_{k i}}\right)+\alpha\left(\frac{M B_{\text {Total }_{-} k i}+M B_{\text {Intra_ki }}}{M B_{\text {Total_ki }}}\right)
\end{aligned}
$$

where $S G T_{k i}$ and $S G S_{k i}$ represent the estimated significance grades of $\mathrm{VP}_{k i}$ from the temporal and spatial domain, respectively. Regarding the $S G T_{k i}$, this study assumes that the cumulative error propagation of succeeding video frames of $\mathrm{VP}_{k i}$ by a geometric progression with common ratio $r=1$. From [7] we find that the error propagation effect strongly depends on the frame position in the GOP, while it is almost independent of the sequence. On the other hand, $S G S_{k i}$ calculates the ratio of the number of intra-refreshed $\mathrm{MBs}$ in $\mathrm{VP}_{k i}$ to the number of total $\mathrm{MBs}$ in $\mathrm{VP}_{k i}$. In this paper, a periodically intra-refresh operation is executed.

In Eq. (1), $\alpha$ is a weighting factor that determines the contributions of $S G T_{k i}$ and $S G S_{k i}$ to $S G_{k i}$. The value of $\alpha$ depends on the complexity of video sequence, which will be discussed in Section 2-2. $N_{I}$ represents the length of a GOP, MB ${ }_{\text {Total_ki }}$ represents the number of $\mathrm{MBs}$ and $M B_{\text {Intra } k i}$ represents the number of intra-MBs in $\mathrm{VP}_{k i}$. The error concealment effect while $\mathrm{VP}_{k i}$ is lost is expressed by

$$
Q_{k i}=\frac{\sum_{j}|P O(j)-P E(j)|^{2}}{\sum_{j}|P O(j)|^{2}}
$$

where $j$ is the pixel index, $P O(j)$ is the original encoded value of pixel $j$, and $P E(j)$ is the recovered value of pixel $j$ when $\mathrm{VP}_{k i}$ is lost and error concealment is activated. The detailed procedure of UPA is shown in Fig. 2. 


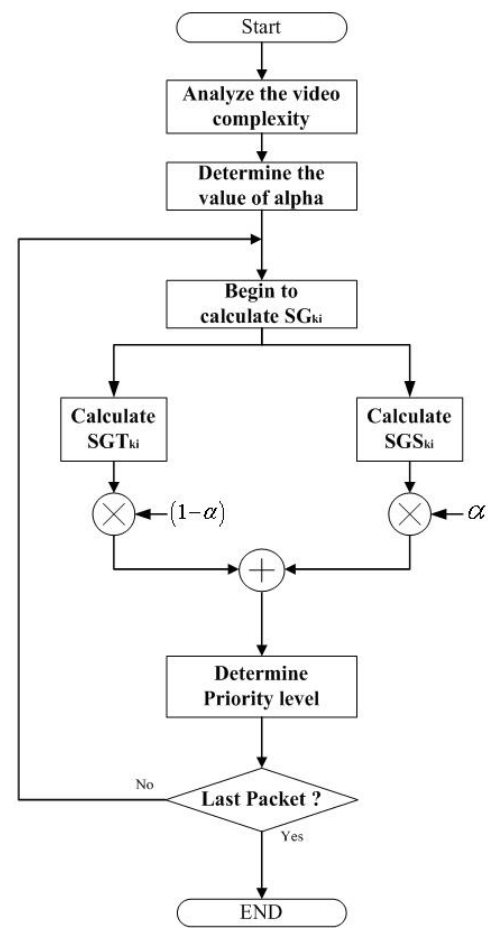

Fig. 2. Detailed operations of UPA

Figures 3 to 5 present the results of executing UPA for "Foreman" sequence, where $\alpha$ is set to $1,0,0.25$, respectively. Figs. 3 and 4 show two extreme cases, that is, Spatial Domain Consideration Only (SDCO) case and Temporal Domain Consideration Only (TDCO) case. Considering the SDCO case that is similar to [6], the height of a vertical line represents the capability of a packet to enhance the received picture quality, as shown in Fig. 3. If the height of a vertical line is explicitly higher than that of adjacent vertical lines, the packet that corresponds to the higher vertical line contains more intra-refreshed MBs and thus provides more contribution to the received quality than other packets. Note that the consideration of error propagation effect is not presented in Fig.3. Regarding the TDCO case that is similar to [5], the height of vertical line gradually decreases when the packet number increases, as shown in Fig. 4. This is mainly because the error propagation resulted from the first erroneous frames of a GOP is larger than that from the later erroneous frames in the same GOP. Note that the content significance of packet cannot be observed in Fig.4.

Regarding the normal case of UPA that is proposed by this paper, the height of a vertical line consists of $S G T_{k i}$ and $S G S_{k i}$, as shown in Fig. 5. The significance grade of a packet does not only depend on the packet content, but also depend on the temporal position of the packet in a GOP. There present two examples in Fig. 5. In the first example, the significance of Packet B is higher than that of Packet A, even the 


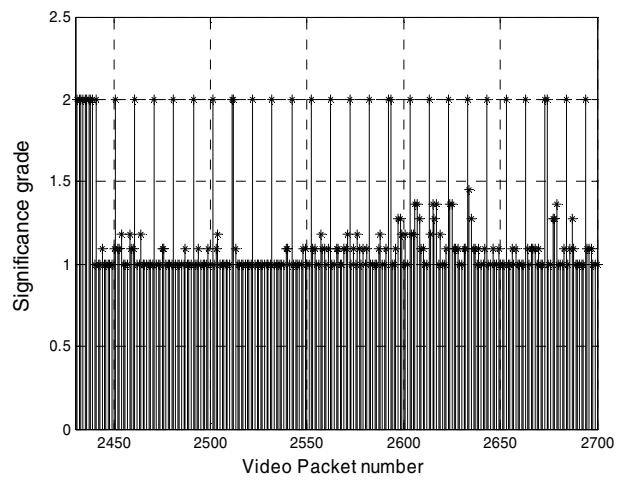

Fig. 3. Results of UPA, $\alpha=1$

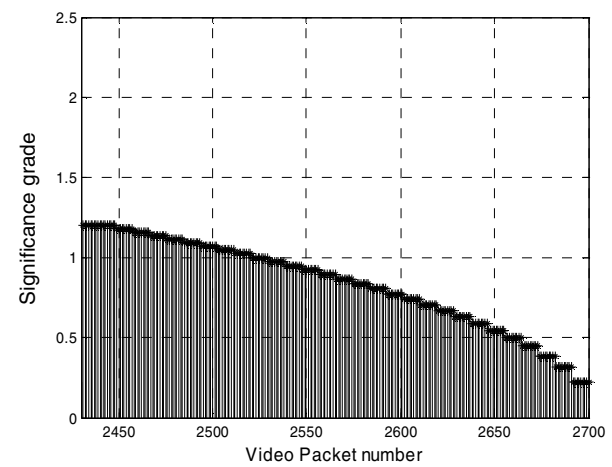

Fig. 4. Results of UPA, $\alpha=0$

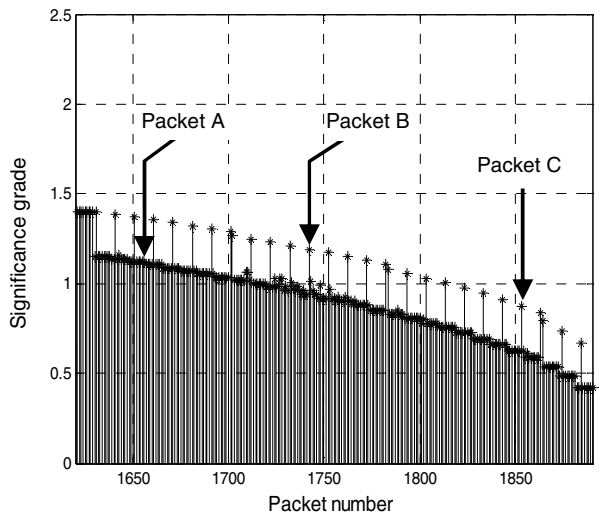

Fig. 5. Results of UPA, $\alpha=0.25$

temporal position of Packet B lag behind Packet A. In the second example, the significance of Packet $\mathrm{C}$ is lower than that of Packet $\mathrm{A}$, even the value of $S G S_{k i}$ of Packet $\mathrm{C}$ is larger than that of Packet $\mathrm{A}$. The capability that estimates the significance 
of packets from both the temporal and spatial domains is the major contribution of this study, which can provide more accurate judgment of packet importance than other traditional methods.

\subsection{Determining Weighting Factor $\alpha$}

Considering a video sequence with low motion variation and low complexity, the similarity between adjacent video frames is high and the error propagation effect in the temporal domain is thus low. Therefore, it is intuitive that the contribution of $S G T_{k i}$ to $S G_{k i}$ should be lower than that of $S G S_{k i}$. Based on the similar concept, UPA flexibly adjusts the weighting factor $\alpha$ between $S G T_{k i}$ and $S G S_{k i}$ according to the video complexity. Referring to common classification of video sequence, this study defines that Class A video sequences have low motion variation and low complexity such as Akyio, Class B video sequences have high motion variation and low complexity such as Foreman, and Class $\mathrm{C}$ video sequences have high motion variation and high complexity such as Stefan.

Figure 6 shows error propagation results of video sequences that belong to different classes, where some video packets of the first $N$ frames in a GOP are assumed to be lost. Observing on Fig. 6, the error propagation of Stefan is serious because of its high motion variation and high complexity. Therefore, the temporal position of the lost packet is very important to the error propagation effect of Class $\mathrm{C}$ video sequences. In contrast, considering the "AKyio", the error propagation effect is not obvious. Therefore, the weight of $S G T_{k i}$ should be lower than that of $S G S_{k i}$ in (1) while considering Class A video sequences. This paper summarizes the above observations and determines various ranges of $\alpha$ for different video classes, as presented in Table 1.

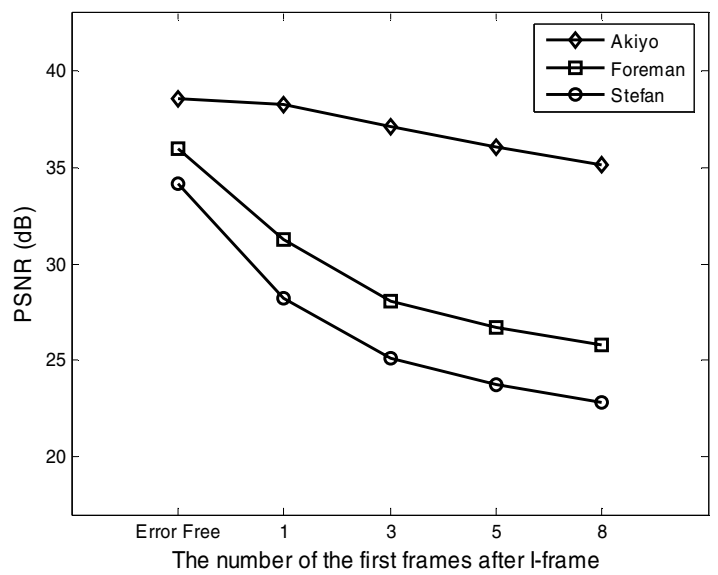

Fig. 6. Error propagation effect of different videos 
Table 1. Range of $\alpha$ for different video classes

\begin{tabular}{cc}
\hline Video Class & Value of $\alpha$ \\
\hline Class A & $0.9 \sim 0.6$ \\
Class B & $0.6 \sim 0.4$ \\
Class C & $0.4 \sim 0.1$ \\
\hline
\end{tabular}

\section{Simulation Results and Discussions}

In this section, the performance of UPA is evaluated by simulations. This paper uses Network Simulator version 2 (NS-2) to simulate the Diffserv network. In these simulation cases, this study uses the H.264 codec and compresses videos at a target rate of $750 \mathrm{~K}$ bps. The video format is CIF and the frame rate is 30 frames per second. In addition, the length of GOP is set to 30 frames and the cyclic intra refreshment function is activated. As shown in Fig.7, video flows have to compete with background flows and the bottleneck link varies its bandwidth dynamically. Three differentiated service levels are provided in the simulated network, where the high priority level, the medium priority level, and the low priority level are named as P1, P2, and P3, respectively. A Weighted Round Robin (WRR) is also utilized here and the Drop Tail operation is activated for queue management.

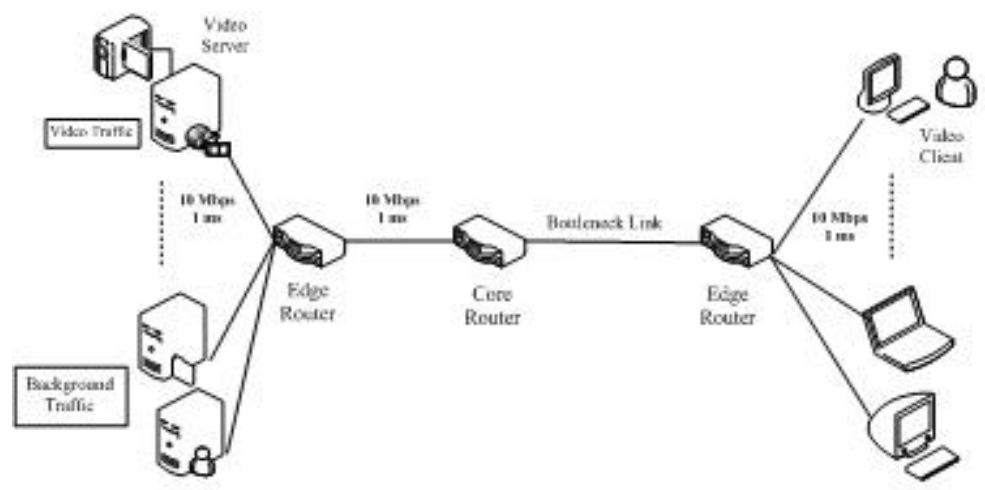

Fig. 7. Simulation architecture using NS-2

Referring to (1), the estimated significance grade of packet is widely distributed from 0 to 2 . Therefore, the mapping between the value of significance grade and the limited differentiated service levels is required, which is called QoS mapping [8]. This study defines three QoS mapping scenarios for different Available Bandwidth (AB) conditions, as shown in Fig. 8. A TCP Friendly mechanism integrated with feedback function is activated in these simulation cases. When the network available bandwidth changes, the video server selects a corresponding QoS mapping scenario based on rules presented in Fig. 8 . 
Figure 9 shows performance comparisons of UPA with TDCO and SDCO methods, where the "Foreman" sequence is used. The TDCO scheme with $\alpha=0$ considers the significance of packet from the temporal domain only and the SDCO method with $\alpha=1$ considers the significance of packet from the spatial domain only. Considering the normal case of UPA, the value of $\alpha$ is set to 0.5. From Fig. 9, it is obvious that the received PSNR using UPA is better than that using TDCO or SDCO. This is mainly because UPA does not only refer to the packet content but also refer to the temporal position of the packet in a GOP. Using UPA, the improvement to received PSNR is significant when the packet loss rate increases. In addition, the received picture quality degraded significantly if the network only provides besteffort delivery quality to video data. Figure 10 shows the results while delivering the "Stefan" sequence. Note that the value of $\alpha$ in the normal case of UPA is changed to 0.7 since the "Stefan" sequence is classified as Class C Video.
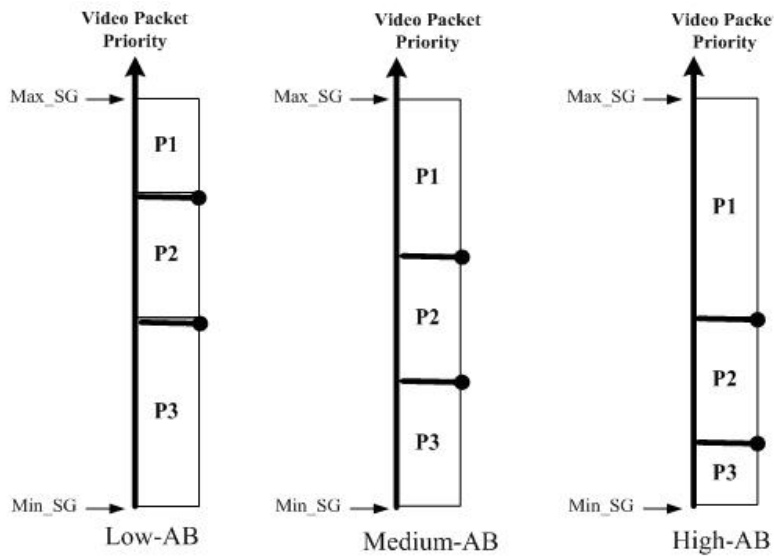

Fig. 8. QoS mapping between SG and differentiated service levels

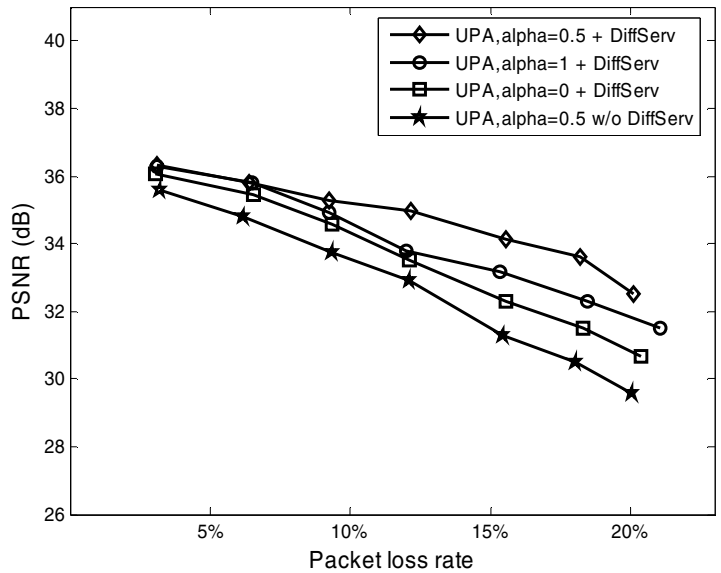

Fig. 9. Performance comparison of UPA with TDCO and SDCO, where Foreman is used 


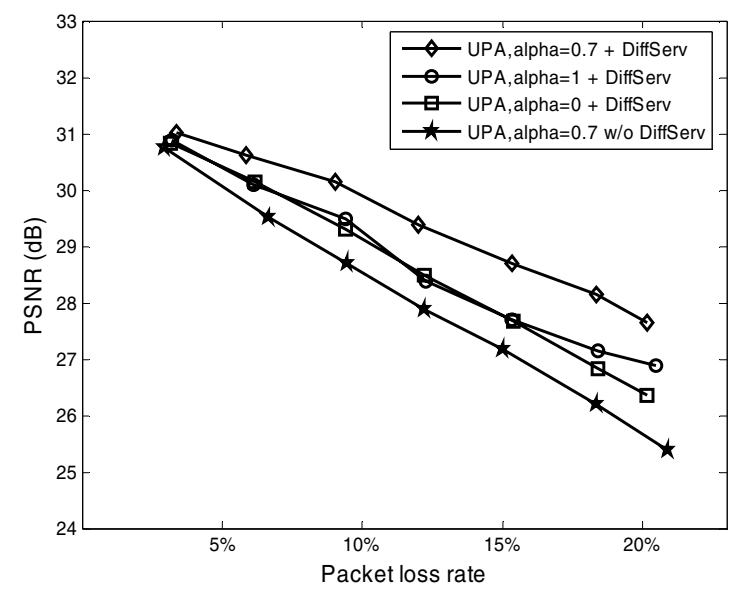

Fig. 10. Performance comparison of UPA with TDCO and SDCO, where Stefan is used

Finally, this paper has tried to include more characteristics of H.264, such as the data partition, into the consideration of UPA. However, we found that the average payload length of video packet decreases significantly, after classifying the significance of video data by many characteristics of H.264. This phenomenon significantly increases the packet header overhead and reduces the overall delivery performance. From the packetization viewpoint, the tradeoff between the error resilience functions of H.264 and the introduced header overhead is an open issue for further study.

\section{Conclusions and Future Works}

When estimating the significance of video packets, the evaluation from the temporal domain only or the spatial domain only is not sufficient. The UPA mechanism proposed by this paper does not only refer to the packet content from the spatial domain, but also refer to the temporal position of the packet in a GOP from the temporal domain. Simulation results show that delivering video data with UPA on differentiated service network outperforms traditional temporal-based-only and spatial-based-only priority strategies up to $1.8 \mathrm{~dB}$ and $1 \mathrm{~dB}$, respectively. In the future, this study will target at the optimization works to the determination of $\alpha$ based on the video characteristics and to the QoS mapping between the significance grade and the limited differentiated service levels in network.

Acknowledgments. The authors would like to thank C. H. Chuang for his precious assistances during the simulation process and his valuable comments for this study.

\section{References}

1. Braden.R., Clark.D., Shenker.S.:Integrated Services in the Internet Architecture: an Overview. RFC 1633, June (1994).

2. Blake.S., Black.D., Davies.E.:An Architecture for Differentiated Services. RFC2475, Dec. (1998). 
3. Magalhaes.J., Guardieiro.P.: A New QoS. Mapping for Streamed MPEG Video over a DiffServ Domain. Communications, Circuits and Systems and West. Sino Expositions, IEEE 2002 International Conference on Volume 1, July (2002) 675-679.

4. C.-H.Ke., C.-K.Shieh., W.-S.Hwang, Ziviani.A.:A Two-Markers System for Improved MPEG Video Delivery in a DiffServ Network. IEEE Communications Letters, IEEE Press, v. 9, April (2005) 381-383.

5. Zhang.F., Pickering.M.R., Frater.M.R., and Arnold.J.F.: Optimal QoS mapping for streaming video over Differentiated Services networks. Acoustics, Speech, and Signal Processing, 2003. Proceedings.2003 IEEE International Conference on Volume 5 (2003).

6. Cote.G., Kossentini.F.:Optimal Intra Coding of Blocks for Robust Video Communication over The Internet. Signal Processing: Image Communication, Sep. (1999) 25-34.

7. Fabio De Vito, Davide Q., Juan Carlos De Martin: Model-based distortion estimation for perceptual classification of video packets. IEEE 6th Workshop on Multimedia Signal Processing (2004).

8. Shin.J., Kim.J., C.-C.J.Kuo:Quality-of-Service Mapping Mechanism for Packet Video in Differentiated Services Network. IEEE Transactions on Multimedia, Vol. 3, No. 2, June (2003) 219-231. 